\title{
Paul Ricoeur y la búsqueda del reconocimiento ${ }^{* \dagger}$ \\ Paul Ricoeur's search for recognition
}

\author{
Edwin Alonso Vargas Bonilla \\ Universidad del Quindío - Colombia
}

$\Phi$

\section{Resumen}

El siguiente texto procura recorrer de manera sucinta el camino filosófico transitado por el pensador francés Paul Ricoeur en su búsqueda de significación para el concepto de reconocimiento. Se abordará el aporte de Ricoeur bajo la siguiente pregunta problema: icómo superar el vacío del término reconocimiento en el ámbito filosófico? Para ello, se presentarán tres momentos: la identidad como transfondo, el desarrollo de la idea y su final significación filosófico-política. Este camino se constituye en una propuesta alternativa que pretende superar el vacío en el que ha caído dicho término a lo largo de la historia de las ideas, abriéndole la puerta al desarrollo de una teoría filosófica del reconocimiento con estatus independiente.

Palabras clave: Reconocimiento, vacío, identidad, ipseidad, mismidad.

\footnotetext{
"Recibido: 30 de marzo de 2020. Aceptado: 14 de mayo de 2020.

Este artículo es producto del trabajo de grado Aproximación al concepto de reconocimiento en Paul Ricoeur, sustentado y aprobando en Junio de 2009.

Contacto: edwin_vargas82@hotmail.com. Profesional en filosofía de la Universidad del Quindío, Magíster en Literatura de la Universidad Tecnológica de Pereira y doctorando en Literatura de la misma Alma Máter. Docente del programa de Licenciatura en Literatura y Lengua Castellana de la Universidad del Quindío.
} 


\begin{abstract}
The following article makes a brief course into de philosophical paths followed by the french thinker Paul Ricoeurs as he searchs for the meaning of the concept of recognition. The guiding question to value the contribution of Ricoeur is: how to surpass the emptyness of the concept of recognition in the philosophical scope? Three moments will be explored to fullfil this purpose: identiy as a background, the development of its idea, and is final political and philosophical meaning. This path offers an alternative proposal to surpass the empty space given tho this term along the history of ideas, opening a way to the development of a philosophical theory of recognition with an independent status.
\end{abstract}

Keywords: Recogntion, Emptyness, Identity, Ipseity, Selfness.

\title{
(C) $(1) \Theta$
}

Material publicado de acuerdo con los términos de la licencia Creative Commons AttributionNonCommercial-NoDerivatives 4.0 International (CC BY-NC-ND 4.0). Usted es libre de copiar o redistribuir el material en cualquier medio o formato, siempre y cuando dé los créditos apropiadamente, no lo haga con fines comerciales y no realice obras derivadas. 


\section{El reconocimiento como problema}

Durante siglos, el reconocimiento, como categoría filosófica, ha estado relegado en el interior del desarrollo de las diversas teorías del conocimiento como una cuestión aleatoria, discontinua. No se le ha tratado con la dignidad filosófica que merece una cuestión que hoy por hoy juega un papel determinante en las relaciones humanas. Sin embargo, dicho concepto en la actualidad asume una fuerte significación desde el punto de vista político, ya que muchas de las determinaciones personales e institucionales tomadas con criterios de identidad han logrado favorecer el reconocimiento de unos y limitar el de otros, generando una brecha que ha tratado de cerrarse históricamente por la vía de la lucha, que en la mayoría de los casos se ha manifestado por medio de la violencia. Para afrontar dicha cuestión Ricoeur explora, tomando como punto de partida un análisis lexicográfico, el ámbito de los vacíos entre las diversas nociones de reconocimiento con miras a la posibilidad de establecer una teoría sobre éste:

el filósofo puede encontrar un estímulo del lado del lexicógrafo en la búsqueda de los pilares -hemos hablado de implícito, de no dicho- que garantizan la transición de una definición a otra. Incumbe a estos pilares, a la vez, crear y superar las desviaciones ocultas bajo la apariencia de una generación continua de las significaciones nuevas a partir de las precedentes. Vamos a intentar un trabajo parecido, sobre lo implícito y lo no dicho en el plano conceptual, con la esperanza de compensar el efecto primero de dislocación producido por la problematización, por un efecto de concertación entre filosofemas que el trabajo sobre las transiciones ha hecho consonantes (Ricoeur 2005 33).

En tal sentido cobra vigencia no sólo el problema tratado, sino también el autor abordado: el filósofo contemporáneo francés Paul Ricoeur (1913-2005). Este asume el reconocimiento como una cuestión que ha sido ampliamente tratada en los círculos lexicográficos, pero poco desarrollada en el ámbito filosófico, en especial en el plano de las relaciones humanas y políticas. Por ello, en su obra Caminos del Reconocimiento (2005), propone una elevación gradual del término desde la dependencia a lo cognitivo a 
la independencia filosófica conceptual, abriéndole sendas para desarrollos posteriores y superar así lo que aquí se denominará un vacío filosófico de no significación.

De esta manera, tomando en cuenta la relevancia actual del concepto y la correspondencia del autor con éste, la pretensión del estudio radica en dar cuenta de la conceptualización del término "reconocimiento", utilizado frecuentemente en el argot filosófico, pero que, por su falta de contenido conceptual no pasa de ser un término vacío que va y viene en el discurso. Para tal empresa, se propone realizar un estudio del concepto expuesto por Ricoeur en Caminos del Reconocimiento, asumiendo como transfondo de dicho problema la idea de identidad expuesta en Sí mismo como otro (2003), al igual que el tratamiento del concepto en sus momentos claves: el reconocimiento de sí y el reconocimiento mutuo, para que, finalmente, observando la significación filosófico-política que gana dicho concepto, se muestre un nivel de superación del vacío teórico de la idea.

En Caminos del Reconocimiento, Ricoeur presenta el resultado de tres conferencias a las que se añaden nuevas elaboraciones. La motivación de la obra se impulsa en el sentimiento de perplejidad frente a la ausencia de significación del término "reconocimiento" en el discurso filosófico, evidenciándose esto en la inexistencia de una sola teoría filosófica del reconocimiento, en contraste con las múltiples teorías del conocimiento. Este vacío contrasta con la coherencia del mismo en el plano lexicográfico: éste puede figurar en un diccionario cualquiera de lengua francesa como "una unidad léxica única a pesar de la multiplicidad" (Ricoeur 2005 11).

Es decir, en el término reconocimiento confluyen varias acepciones semánticas desarrolladas por los lexicógrafos. Ante tal posibilidad lexicográfica y riqueza polisémica de la palabra ipor qué la tradición filosófica no ha prestado al reconocimiento la atención que merece como idea digna de desarrollo conceptual? También se puede plantear dicho problema, según palabras del propio Ricoeur, en los siguientes términos: "es un hecho que no existe teoría digna de éste nombre a modo como existen una o varias teorías del conocimiento. Esta sorprendente laguna contrasta con el tipo de coherencia que permite al término reconocimiento figurar en un diccionario como unidad léxica a pesar de la multiplicidad" (Ricoeur 2005 11). 
La propuesta de Ricoeur frente a este problema es una apuesta para "conferir a las diversas apariciones filosóficas conocidas del término reconocimiento la coherencia de una polisemia regulada, capaz de proporcionar la réplica a la del plano léxico" (Ricoeur 2005 12). En este sentido, el propósito del filósofo es realizar un estudio partiendo de la riqueza lexicográfica del término reconocimiento para elevarse a un desarrollo filosófico de éste que le separe gradualmente del reducido plano cognitivo al que ha sido confinado y le confiera un estatuto filosófico, político y ético independiente que le abra "caminos" para un posterior y creciente desarrollo conceptual.

¿Cómo pretende sacar adelante tal pretensión el filósofo en cuestión? Su hipótesis de trabajo la resume en lo siguiente: "la dinámica que pone en movimiento la investigación consiste en invertir en el plano mismo de la gramática del verbo reconocer, su uso en la voz activa a su empleo en la voz pasiva: reconozco activamente algo, a personas, a mí mismo; pido ser reconocido por los otros" (Ricoeur 2005 12). Así, la estrategia utilizada por el autor indica un paso gradual del término reconocimiento de la dependencia al plano epistemológico hacia la autonomía conceptual en el plano filosófico-político para lograr su independencia teórica.

Teniendo en cuenta lo anterior, a saber, el problema, el propósito y la metodología de Ricoeur, se pueden observar tres cuestiones que confluyen en el asunto:

1. Cuando se habla de reconocimiento, ise habla de reconocimiento de qué? De esta manera, la cuestión de la identidad aparece como transfondo permanente del concepto.

2. Cuando se habla del paso del término reconocimiento de la voz activa a la pasiva, es decir, de un estar ligado a lo cognitivo a una independencia filosófica que le abra caminos a su desarrollo teórico, icómo se logra? Aquí entran en juego dos momentos que Ricoeur puntualiza como el reconocimiento de sí y el reconocimiento mutuo.

3. Cuando el autor propone que el término reconocimiento asuma un estatus filosófico independiente, ¿qué significación toma dicho concepto? Es ahí cuando la significación filosófico-política del concepto sale a flote.

Las problemáticas cuestiones anteriormente anotadas frente a los planteamientos de Ricoeur pueden subsumirse en una pregunta, de igual modo problemática: ¿de qué manera reconocimiento pasa de ser un término disperso, ligado a lo cognitivo para convertirse en un concepto filosófico independiente que posibilite el desarrollo de una 
teoría filosófico-política del reconocimiento?, o, dicho en otras palabras: ¿cómo superar el vacío del término reconocimiento en el ámbito filosófico?

\section{El reconocimiento como concepto}

Inicialmente se puede preguntar: al hablar de reconocimiento, ise hace referencia al reconocimiento de qué? De este modo, como idea general, se tiene presente que para realizar un estudio del concepto de reconocimiento siguiendo a Ricoeur es necesario, como transfondo, abordar la cuestión de la identidad. Así, la identidad -el qué del reconocimiento- aparece presente en todos los momentos de desarrollo de dicha idea.

Pero, icómo involucrar la cuestión de la identidad con el concepto de reconocimiento? Frente a esto, Ricoeur alude a la ruptura entre el cogito planteado por Descartes y el cogito quebrado por Nietzsche. En ese sentido, se puede intuir que la identidad como posibilidad de reconocimiento se forja entre el ejercicio filosófico de superación del sujeto fundacional de la modernidad, enunciado como "yo", y la propuesta contemporánea de un sí reflexivo.

Esta revisión a las filosofías del sujeto empieza por observar el motivo por el cual el "yo" se fortalece filosóficamente, de tal manera que se sustenta y conserva como el problema central de la filosofía durante toda la modernidad. Ese motivo es la pretensión de hacer del "yo" el fundamento último. Ambición que -señala Ricoeur- "se ha radicalizado desde Descartes a Kant, después de Kant a Fichte y, finalmente, al Husserl de las Meditaciones Cartesianas" (2003 XV). Tal cogito es un yo sistemático que actúa como fundamento epistemológico, pero que está distanciado de las relaciones políticomorales. Ricoeur es crítico precisamente de esas filosofías del yo inmersas en lo epistemológico pero alejadas de lo político, las cuales se mantuvieron firmes durante varios siglos.

Previo al desarrollo de la idea de identidad, Ricoeur introduce un estudio crítico del yo, en el que, de entrada, propone que el cogito moderno ha sido ya superado por las nuevas propuestas de la subjetividad. Desde una actitud crítica, Ricoeur investiga acerca 
del contenido de ese sujeto, pues la idea cartesiana del cogito plantea la existencia de un yo formal que cumple con la función de dudar, y que, se supone, en tanto duda piensa; pero frente a éste no se ha realizado el ejercicio de responder a la pregunta iqué es ese yo? Lo que pretende Ricoeur con el estudio del concepto de identidad es, precisamente, no caer en el mismo error categorial cartesiano y darle un contenido a la identidad a la cual se refiere el concepto de reconocimiento, sin antes entrar a desarrollar éste último.

Para Descartes, el cogito es el yo formal que se reduce al acto de pensar, cuyo correlato es la idea de dios: el yo es posible en la medida en que ese dios epistémico lo permite. Ante la idea de un dios perfecto, veraz, infinito, ilimitado, aquel yo se asume a sí mismo como imperfecto, dubitable, finito, limitado: en este caso, siguiendo a Ricoeur, tenemos un primer indicio de reconocimiento epistemológico, en el que la identidad del yo se encuentra vacía.

En ese tránsito del yo a la identidad, partiendo de Nietzsche, se señala la función del lenguaje en la crítica a las filosofías modernas del sujeto basadas en un yo fundacional que es objeto de la crítica nietzscheana. En efecto, en el prólogo a La genealogía de la moral se evidencia esta problemática con una frase breve y precisa: "Nosotros los que conocemos somos desconocidos para nosotros" (Nietzsche 2001 21). Mientras que en Aurora indica que

no hay nada que resulte más difícil de conocer al hombre, desde los tiempos más remotos hasta hoy, que el desconocimiento que tiene de sí mismo, y no sólo respecto al bien y al mal, sino también respecto a algo mucho más esencial (Nietzsche 2000 133).

Al respecto, Ricoeur nos recuerda que, para el filósofo de Basilea, esos grandes supuestos que se han establecido como piedra de toque para erigir los grandes sistemas filosóficos modernos no son otra cosa que artificios del lenguaje que, usados por los filósofos, han referido ciertas ideas que fundamentan la estructura de esos enormes edificios teóricos. Para tal efecto, se remonta a algunos escritos contemporáneos al Nacimiento de la Tragedia, específicamente al Curso de Retórica (Basilea, 1872-1873) y Verdad y mentira en el sentido extra moral (1873), en los que Nietzsche hace una crítica a la pretensión de la filosofía de convertirse en ciencia fundacional (Cit en Ricoeur 2003 XXIII). 
La crítica nietzscheana a la pretensión de la filosofía moderna de erigirse en ciencia del fundamento parte del papel que juega el lenguaje en la filosofía. Efectivamente, se lee en el Crepusculo de los ídolos: "La razón en el lenguaje: iOh, que vieja mujerzuela engañadora! Temo que no vamos a librarnos de Dios porque continuamos creyendo en la gramática..." (Nietzsche 2016 633). En ese sentido, Nietzsche muestra que las filosofías de la subjetividad han relegado de la reflexión filosófica el papel que juega el uso del lenguaje en la argumentación que sustenta al cogito, haciendo públicas las estrategias retóricas usadas para tal fin de manera oculta; estrategias a las que no se ha dado aceptación en el plano del discurso, excusándose en la inmediatez de la reflexión.

Dicha reducción de las ideas fundacionales modernas a tropos del lenguaje constituyen lo que el filósofo francés señala como la interpretación retórica a esas filosofías del sujeto fundacional; crítica que tal vez, de un modo temerario, puede asumirse como la interpretación de los conceptos fundacionales -entre estos el cogitocomo palabras utilizadas para referir ficciones, entidades metafísicas inexistentes y que, por tanto, tal lenguaje filosófico resulta ficcional y mentiroso. Siendo ésta la característica del lenguaje por medio del cual se ha hecho la filosofía en la modernidad, no puede esperarse cosa distinta de la filosofía resultante del mismo.

Esto le abre paso al desarrollo de nuevas propuestas filosóficas no sistemáticas en las que el lenguaje ya no refiere supuestos incólumes o piedras angulares para grandes estructuras teóricas, sino que da apertura a la multiplicidad de interpretaciones y posibilidades del pensamiento. A esa línea no fundacional, pero sí interpretativa, se puede asociar a Paul Ricoeur y su trabajo filosófico respecto a la cuestión abordada. Sus interpretaciones en ese ámbito abordan la dialéctica entre el reconocimiento de sí y el reconocimiento del otro como el campo de estudio que le da gradualmente autonomía conceptual al término reconocimiento desligándolo poco a poco de las funciones meramente cognitivas a las que se hallaba confinado en las múltiples teorías del conocimiento, dándole estatus filosófico independiente.

En ese sentido, el trabajo hermenéutico de Ricoeur apunta a la superación del vacío filosófico del concepto, abriéndole posibilidades a su desarrollo teórico. Esto último obedece a la propuesta ricoeuriana de aliviar el tan marcado contraste entre la no existencia de una teoría del reconocimiento frente a las múltiples teorías del 
conocimiento desarrolladas desde la modernidad. Vemos, entonces, que nuestro filósofo aprovecha la ausencia de desarrollo filosófico de las acepciones del término reconocimiento y evidencia un nuevo campo de investigación que resulta interesante para la contemporaneidad.

\section{El reconocimiento como identidad y lucha}

Como se dijo anteriormente, la crítica nietzscheana a las filosofías del sujeto y la duda hiperbólica aplicada a los supuestos fundacionales de tales filosofías rompen con el cogito moderno planteado desde Descartes. De esta manera, se inaugura una nueva posibilidad filosófica: la subjetividad como interpretación. Este es el punto de partida de Ricoeur, desde el cual hace el ejercicio de deconstrucción del sujeto formal para plantear una nueva identidad personal.

La superación de la noción de reconocimiento relegado a la función cognitiva de identificación se inicia poniendo en escena al qué del reconocimiento, en primer lugar, como algo indeterminado: hay algo que se reconoce, pero que aún no se tiene claro qué es; por lo cual, el reconocimiento se da, en primera instancia, como una mera función: la identificación. Posteriormente, al cuestionarse por el qué de tal objeto, éste pasa a ser determinado como aquello que se identifica como igual a sí mismo (reconocimiento de sí). Luego de que el objeto del reconocimiento se identifique como igual a sí mismo, se abre la posibilidad a la relación de reconocimiento del sí mismo con lo otro (reconocimiento mutuo).

Al hablar de identidad personal, Ricoeur tiene en cuenta dos momentos: un primer momento de la identidad que señala que una cosa es igual a sí misma y se reconoce a sí misma como tal, a la cual llama identidad idem; y otro que le permite a esa identidad idem ser cambiante con el paso del tiempo sin perder la estructura inicial de la mismidad: a ese momento se refiere con el nombre de identidad ipse o ipseidad. A esta segunda dimensión de la identidad Ricoeur la llama, también, identidad narrativa. Es decir, la identidad personal tiene dentro de sí la dimensión narrativa y es precisamente el tiempo el que permite la relación entre ambas nociones de identidad. 
Se puede decir, por ello, que dentro del desarrollo del concepto de identidad personal se encuentra inmersa la cuestión de la temporalidad: en el paso de la identidad idem (mismidad) a la identidad ipse (ipseidad, otreidad), el tiempo juega su papel como factor regulador de la dialéctica dentro de la cual se dota de contenido a la identidad. A partir de esto, reconocer algo que se da dos o más veces como lo mismo constituye la primera instancia de identidad (mismidad) que puede considerarse un primer momento en el desarrollo del concepto de reconocimiento: el reconocimiento como identificación. Sin embargo, hay una cuestión que éste reconocerse a sí mismo como idéntico a sí necesita franquear: ¿cómo permanece en el tiempo?, y, como no sólo se está hablando de la estructura del sí, sino además de su contenido, ¿qué es lo que permanece en el tiempo? Ante ésta pregunta Ricoeur alude al carácter y a la palabra dada.

El carácter -nos dice Ricoeur- es una disposición adquirida en el tiempo y que permanece en éste a la manera que la costumbre aristotélica (ethos) por su uso constante se hace hábito y termina configurando el carácter (éthos) del individuo. Pero, al interior de ese éthos no existiría aún una frontera clara entre la mismidad y la otreidad, ya que dicha posibilidad de ser otro formaría parte constitutiva de ese sí mismo. En este caso, el qué o contenido del sí mismo es la identidad en el carácter adquirido.

Por otra parte, la palabra dada afirma la mismidad del carácter y se convierte en un desafío al tiempo. Cumplir con la palabra dada en algún momento por el sí mismo requiere de la conservación de la estructura del carácter en la memoria a pesar de los posibles cambios en el tiempo. Por eso, en la palabra dada la ipseidad y la mismidad encuentran su punto máximo de diferenciación, ya que los cambios introducidos en el sí mismo por el tiempo se regulan de manera tal que la promesa hecha permanece en el sí mismo para su cumplimiento.

Tal es el contenido de la identidad personal en Ricoeur: un carácter que tiene dentro de sí las posibilidades de ser sí mismo al conservar los rasgos constitutivos de su personalidad y, a su vez, ser otro por medio del cambio que deviene en el transcurrir del tiempo, sin perder la estructura inicial que lo identifica. En este sentido, al darle al qué del reconocimiento un contenido conceptual se tiene un primer momento fuerte de superación del vacío de no significación del reconocimiento, propuesto desde el principio. 
Con miras a continuar con la superación de dicho vacío, la apuesta ricoeuriana consiste, en segundo lugar, en abrir el campo de investigación para desarrollar una teoría del reconocimiento con independencia conceptual a las teorías del conocimiento. Para esto, su hipótesis de trabajo se sintetiza en el cambio de la voz activa (reconozco) a la voz pasiva (soy reconocido, pido ser reconocido) del término reconocer, que devela un paso gradual del término reconocimiento del campo de lo intelectivo a lo político. Es evidente que el filósofo en cuestión busca dotar de dignidad filosófica al concepto tratado, ya que si bien éste ha hallado cabida en los círculos filosóficos y políticos actuales, adolece de la falta del desarrollo teórico propio de una idea de tan profunda trascendencia.

Ante tal ausencia, Ricoeur explota el campo lexicográfico a favor de la investigación teórica y explora entre los vacíos de las distintas acepciones del término reconocer las posibilidades de conceptualización para una idea filosófica de reconocimiento. Además, ya ubicado el término en un campo conceptual, recurre al estudio de una noción política como lo es la idea de la lucha por el reconocimiento, que representa el tercer momento de superación del vacío conceptual planteado como problema de investigación. Este viraje de la cuestión pone sobre la mesa un interesante diálogo contemporáneo entre Paul Ricoeur y Axel Honeth (1949).

Precisamente, es la idea de lucha por el reconocimiento la que nos sumerge en una teoría política que representa la actualidad de la cuestión problemática rastreada en éste trabajo. Frente a esto, Ricoeur, interlocutando con Honeth, señala que la lucha por el reconocimiento ha sido impulsada y es impulsada en la actualidad histórica y moralmente por los modelos de negación del reconocimiento. El planteamiento consiste en que, ante la negación de reconocimiento en ciertos ámbitos, lo que se presenta es una lucha por reivindicarlo.

En ese orden de ideas, se exponen tres modelos de negación y de lucha por el reconocimiento: un reconocimiento prejurídico, denominado como la lucha por el reconocimiento y el amor; un reconocimiento jurídico, que pone en perspectiva la alteridad entre el juego jurídico de los derechos y las responsabilidades; y, finalmente, la lucha por el reconocimiento y los estados de paz, en el que se enfrentan los estados de 
justicia y de paz como contrarios, pero reconciliables, en el juego del don y el contradon.

Al final de la lucha por el reconocimiento en cada uno de los tres planos antes señalados se reivindica la dignidad de aquellos a los que les ha sido negado el reconocimiento. Es decir, ni la dignidad ni el reconocimiento aparecen antes de recorrer el camino de la lucha por su reivindicación, por lo que ésta se ha hecho y se sigue haciendo presente en las distintas sociedades. De esta manera, se puede concluir que la lucha por el reconocimiento aún no termina y tiende a perpetuarse en el tiempo debido a la disimetría entre la igualdad de derechos y la desigualdad de acceso a los recursos y oportunidades. Por esta razón, Ricoeur propone los estados de paz como contextos alternativos a los estados de violencia, para que las luchas por el reconocimiento se desarrollen por la vía de las motivaciones morales y políticas tendientes a la búsqueda de una sociedad que aprenda a superar sus disyuntivas internas pacíficamente.

Finalmente, luego de exponer el recorrido de Ricoeur en la configuración de la noción estudiada, que toma como punto de partida el abordaje de ésta en tanto problema que se afronta ante la inexistencia de una teoría que le otorgue un estatuto epistemológico propio; pasando por la exploración conceptual desde las filosofías del sujeto fundacional moderno (Descartes) y su negación (Nietzsche); hasta llegar a las nociones de identidad personal e identidad narrativa que le abren paso a la lucha por el reconocimiento; se puede concluir que, por medio de la visión ricoeuriana, se abren sendas para superar el vacío de no significación de dicha idea, ya que se evidencia en su desarrollo una elevación gradual desde un término confinado a las teorías del conocimiento, hasta ganar contenido teórico como concepto que abre caminos a la construcción de una teoría independiente.

\section{Referencias}

Descartes, René. Discurso del método. Madrid:Tecnos, 2003.

Descartes, René. Meditaciones metafísicas. Madrid: Gredos, 1987. 
Honneth, Axel. La lucha por el reconocimiento: por una gramática moral de los conflictos sociales. Barcelona: Crítica, 1997.

Honneth, Axel y Fraser, Nancy. ¿Redistribución o reconocimiento? Un debate políticofilosófico. Madrid: Ediciones Morata, 2006.

Nietzsche, Friedrich. La genealogía de la moral. Madrid: Alianza, 2001.

Nietzsche, Friedrich. El nacimiento de la tragedia. Madrid: Alianza, 2014.

Nietzsche, Friedrich. Aurora. Pensamientos sobre los prejuicios morales. Madrid, Biblioteca Nueva, 2000.

Nietzsche, Friedrich. Obras completas IV. Escritos de madurez. Madrid: Tecnos, 2016

Ricoeur, Paul. Sí mismo como otro. Traducción de Agustín Neira Calvo. México: Siglo XXI, 2003

Ricoeur, Paul. Caminos del Reconocimiento. Tres estudios. Madrid: Trotta, 2005. 